\title{
A decade of stability for wMel Wolbachia in natural Aedes aegypti populations
}

Perran A. Ross ${ }^{1}$, Katie L. Robinson ${ }^{1}$, Qiong Yang ${ }^{1}$, Ashley G. Callahan ${ }^{1}$, Thomas L. Schmidt ${ }^{1}$, Jason K. Axford $^{1}$, Marianne P. Coquilleau ${ }^{1}$, Kyran M. Staunton ${ }^{2}$, Michael Townsend ${ }^{2}$, Scott A. Ritchie ${ }^{2}$, Meng-Jia $\mathrm{Lau}^{1}$, Xinyue $\mathrm{Gu}^{1}$, Ary A. Hoffmann ${ }^{1}$

1. Pest and Environmental Adaptation Research Group, Bio21 Institute and the School of BioSciences, The University of Melbourne, Parkville, Victoria, Australia. 2. Australian Institute of Tropical Health and Medicine, James Cook University, Smithfield, Queensland, Australia.

\section{Abstract}

Mosquitoes carrying Wolbachia endosymbionts are being released in many countries for arbovirus control. The wMel strain of Wolbachia blocks Aedes-borne virus transmission and can spread throughout mosquito populations by inducing cytoplasmic incompatibility. Aedes aegypti mosquitoes carrying wMel were first released into the field in Cairns, Australia, over a decade ago, and with wider releases have resulted in the near elimination of local dengue transmission. The long-term stability of Wolbachia effects is critical for ongoing disease suppression, requiring tracking of phenotypic and genomic changes in Wolbachia infections following releases. We used a combination of field surveys, phenotypic assessments, and Wolbachia genome sequencing to show that wMel has remained stable in its effects for up to a decade in Australian Ae. aegypti populations. Phenotypic comparisons of wMelinfected and uninfected mosquitoes from near-field and long-term laboratory populations suggest limited changes in the effects of wMel on mosquito fitness. Treating mosquitoes with antibiotics used to cure the wMel infection had limited effects on fitness in the next generation, supporting the use of tetracycline for generating uninfected mosquitoes without off-target effects. wMel has a temporally stable within-host density and continues to induce complete cytoplasmic incompatibility. A comparison of wMel genomes from pre-release (2010) and nine years post-release (2020) populations show few genomic differences and little divergence between release locations, consistent with the lack of phenotypic changes. These results indicate that releases of Wolbachia-infected mosquitoes for population replacement are likely to be effective for many years, but ongoing monitoring remains important to track potential evolutionary changes.

\section{Author summary}

Wolbachia are endosymbionts that can block the transmission of arboviruses by mosquitoes. Aedes aegypti mosquitoes carrying the wMel strain of Wolbachia have been released in 'population replacement' interventions, which aim to establish wMel in mosquito populations, thereby reducing their ability to spread disease. Wolbachia population replacement programs began only a decade ago, raising uncertainty about their long-term effectiveness. Here we provide a comprehensive assessment of the long-term stability of wMel from the very first Wolbachia population replacement release. We show that there is no evidence for changes in the phenotypic effects of wMel in mosquitoes, and confirm that the wMel genome has changed very little in the decade since field releases began. wMel remains at high levels within mosquitoes, suggesting that its ability to block virus transmission has been retained. Our data provides confidence that Wolbachia population replacement releases will provide 
ongoing protection against arbovirus transmission.

\section{Introduction}

Open field releases of Wolbachia-infected mosquitoes are becoming one of the best ways to control arbovirus transmission. Wolbachia "population replacement" programs involve the release of mosquitoes carrying a Wolbachia infection that spreads through mosquito populations and reduces their vector competence [1-3]. Several different Wolbachia strains from other insects have been introduced into Ae. aegypti mosquitoes through microinjection, with many of them reducing the ability of mosquitoes to transmit arboviruses including dengue, Zika and chikungunya [4-6]. The establishment of wMel and wAlbB Wolbachia strains in natural populations has suppressed arbovirus transmission by Ae. aegypti in release locations $[7,8]$. Population replacement relies on maternal transmission of Wolbachia as well as cytoplasmic incompatibility between Wolbachia-infected males and uninfected females to drive and maintain the Wolbachia infection into the population. Successful establishment and ongoing persistence depends on properties of the Wolbachia strain as well as local environmental conditions which can influence mosquito dispersal [9], Wolbachia maternal transmission [10], cytoplasmic incompatibility [11] and host fitness effects of Wolbachia [12, 13].

Wolbachia releases for population replacement first took place in 2011, where wMel-infected Ae. aegypti were released in two suburbs of Cairns, Australia: Gordonvale and Yorkeys Knob [14]. The wMel infection rapidly increased in prevalence and has persisted at a high frequency in these suburbs for many years $[15,16]$. Releases of wMel-infected Ae. aegypti have since expanded to cover nearly the entire distribution of Ae. aegypti in Australia $[16,17]$. Following the stable establishment of wMel in almost all release locations, local dengue transmission has almost been eliminated in the country [16, 17].

Releases of wMel-infected Ae. aegypti have now been carried out in several dengue-endemic cities including Yogyakarta, Indonesia [18] and Rio de Janeiro [19, 20] and Niterói [21, 22], Brazil. Quasiexperimental and/or randomized controlled trials show that wMel releases have reduced dengue incidence by $>69 \%[8,22,23]$, with reductions in chikungunya and Zika transmission also apparent in some locations [22]. Long-term monitoring shows that wMel has persisted in Ae. aegypti populations at high frequencies for many years $[16,17,21]$, though in some locations the infection has remained at an intermediate frequency or dropped out, requiring supplemental releases [19, 20, 22]. wMel frequencies can also fluctuate seasonally, likely due to high temperatures experienced in larval habitats [24, 25].

Theory predicts that Wolbachia infections, mosquitoes and viruses may evolve, potentially rendering Wolbachia population replacement less effective over time [26-28]. Previous studies in Drosophila demonstrate the potential for evolutionary changes affecting both the Wolbachia and host genomes. The wRi Wolbachia strain invaded Australian populations of Drosophila simulans and rapidly shifted from inducing a host fitness cost to a host fitness benefit [29]. The wMelPop strain also induced weaker host fitness costs and cytoplasmic incompatibility across time after being transferred to a novel Drosophila host [30,31]. Selection experiments show that shifts in the phenotypic effects of Wolbachia are often due to host genetic changes [32]. So far there have been limited changes observed in Wolbachia genomes across several years after transinfection to novel hosts such as wCer 2 in Drosophila [33] and wMelPop-CLA in Ae. aegypti [34]. 
In mosquitoes, there is a clear distinction between natural Wolbachia infections and novel transinfections, where the latter tends to induce deleterious effects [1]. This suggests that the effects of Wolbachia transinfections may weaken across evolutionary timescales. Since population replacement programs began only a decade ago, there is still limited information on evolutionary changes following deliberate Wolbachia invasions. In laboratory populations of Ae. aegypti, the wMelPop-PGYP strain has continued to induce complete cytoplasmic incompatibility for the last decade, but some fitness costs appear to have weakened [35]. In field populations, the wMel strain has shown stable phenotypic effects, with no evidence for changes in dengue virus blocking [21,36], cytoplasmic incompatibility [15, 37] or effects on fertility [15] after a period of 1 year or more under field conditions. Whole Ae. aegypti [38] and Wolbachia [39, 40] genome sequencing studies show limited genomic changes after wMel has been established in Cairns for at least 7 years.

Given the importance of tracking the long-term stability of Wolbachia infections in mosquito populations, we have now collected additional data on the phenotypic and genomic stability of wMel from the first ever population replacement releases in Cairns, Australia in 2011. Our data from up to a decade post-release show that the phenotypic effects of wMel largely remain stable in both laboratory and near-field Ae. aegypti populations. We also extend the findings of Huang et al. [39] to show limited genomic changes in Wolbachia over the span of a decade, with no divergence in wMel genomes between different release locations. Our data point to the likely long-term effectiveness of Wolbachia population replacement programs globally.

\section{Materials and methods}

\section{Field collections and colony establishment}

Aedes aegypti mosquitoes were collected as eggs from suburban Cairns in 2016, 2018, 2019 and 2020 from ovitraps. Felt strips from ovitraps were collected and processed identically to previously described methods [24]. Ovitrapping was performed throughout suburban Cairns in September-October 2016 (19 suburbs, 50-100 traps per suburb) and February-March 2018 (12 suburbs, 40-100 traps per suburb), many of which were targeted by wMel release programs from 2011 to 2017 [14, 16, 41]. The 2019 and 2020 field collections targeted two suburbs with 20 ovitraps each, Gordonvale and Yorkeys Knob, where wMel-infected Ae. aegypti were released in 2011 [14]. Subsets of Ae. aegypti larvae from central Cairns, Gordonvale and Yorkeys Knob were pooled from all traps within a suburb to establish laboratory populations for phenotypic comparisons. Thirty individuals from the $F_{1}$ and $F_{2}$ generations were screened for Wolbachia infection (see below) to confirm fixation of wMel within the laboratory populations. All populations were maintained at census size of $\sim 450$ individuals per generation at $26^{\circ} \mathrm{C}$ and a 12:12 light:dark cycle as described previously [42]. Female mosquitoes were fed on the forearm of a single human volunteer for egg production.

\section{Wolbachia infection frequency and density}

Wolbachia infection frequencies were estimated from the 2016 and 2018 field collections by screening subsets of individuals hatching from ovitraps for Wolbachia. For the 2016 field collections, life stage was uncontrolled, with a mix of adults and larvae tested, thus data were only suitable for Wolbachia frequency estimates. Up to 10 individuals were screened per trap, with between 10 and 243 individuals 
screened per suburb. For the 2018 field collections, $304^{\text {th }}$-instar larvae from 15 ovitraps ( 2 per trap) per suburb were screened for Wolbachia infection. Larvae were reared at a controlled density (50 larvae per tray) and stored at the same age (5 d post-hatching), allowing for a comparison of Wolbachia density across suburbs. In June 2019, we measured wMel density in adults (15 females and 15 males) from laboratory populations that were established from field collections at different times (Gordonvale at $\mathrm{F}_{1}$, Yorkeys Knob at $F_{1}$ and $F_{16}$, central Cairns at $F_{9}$ and the wMel lab population). Aedes aegypti from field collections and laboratory populations were screened for Wolbachia infection status and density using a Roche Lightcycler 480 according to previously described methods [43]. Genomic DNA was extracted with $250 \mu \mathrm{L}$ of $5 \%$ Chelex 100 Resin (Bio-Rad laboratories, Hercules CA) and $3 \mu \mathrm{L}$ of Proteinase $\mathrm{K}(20 \mathrm{mg} / \mathrm{mL}$ ) (Roche Diagnostics Australia Pty. Ltd., Castle Hill New South Wales, Australia). Tubes were incubated for $60 \mathrm{~min}$ at $65^{\circ} \mathrm{C}$ then $10 \mathrm{~min}$ at $90^{\circ} \mathrm{C}$. Three primer sets were used to amplify markers specific to mosquitoes (mRpS6_F 5'AGTTGAACGTATCGTTTCCCGCTAC3' and mRpS6_R 5' GAAGTGACGCAGCTTGTGGTCGTCC3'), Ae. aegypti (aRpS6_F 5'ATCAAGAAGCGCCGTGTCG3' and aRpS6_R 5'CAGGTGCAGGATCTTCATGTATTCG3'), and wMel (w1_F 5'AAAATCTTTGTGAAGAGGTGATCTGC3' and w1_R 5' GCACTGGGATGACAGGAAAAGG3'). Relative Wolbachia densities were determined by subtracting the crossing point $(\mathrm{Cp})$ value of the wMel-specific marker from the $\mathrm{Cp}$ value of the $A e$. aegypti-specific marker. Differences in $\mathrm{Cp}$ were averaged across 3 consistent replicate runs, then transformed by $2^{\text {n }}$.

\section{Phenotypic comparisons}

We performed two sets of experiments to evaluate the phenotypic effects of wMel derived from field and laboratory populations. In the first set, populations were cured with tetracycline to remove the wMel infection, maintaining similar genetic backgrounds between infected and uninfected lines. In the second set, we used backcrossing to introduce the wMel infection from different origins to a common genetic background, then compared populations to uninfected lines that had been crossed to the same background. Both sets of experiments involved the wMel Lab population which was collected from Cairns in 2014 and had spent at least 60 generations in the laboratory before the first set of experiments commenced. All experiments were performed at $26^{\circ} \mathrm{C}$ and a $12: 12$ light:dark cycle.

Experiment 1 was performed in September 2018 using wMel-infected populations collected from Yorkeys Knob in February 2018 (wMel YK) and Cairns in 2014 (wMel Lab). wMel YK and wMel Lab were divided into four population cages each. Two replicate populations from each line were treated for three consecutive generations with tetracycline hydrochloride $(2 \mathrm{mg} / \mathrm{mL}$ ) provided to adults in $10 \%$ sucrose solution to cure the wMel infection. Females were blood fed at $10 \mathrm{~d}$ old to ensure that they had fed on the antibiotic solution prior to blood feeding. The other two replicate populations from each line were left untreated but reared synchronously. All populations were reared in the absence of antibiotics for two generations before experiments commenced, with thirty adults from each population screened for Wolbachia infection to ensure complete removal of wMel in the treated lines (wMel YK.tet and wMel Lab.tet) and fixation of $w$ Mel in the untreated lines ( $w$ Mel YK and wMel Lab). The wMel YK and wMel YK.tet populations were at $F_{8}$ in the laboratory when experiments commenced.

Experiment 2 was performed in August 2020 using wMel-infected populations collected from Yorkeys Knob in February 2020 (wMel YK), Gordonvale in February 2020 (wMel GV), Cairns in 2014 (wMel Lab), 
as well as an uninfected population that had been cured of wMel in the previous experiment ( $w \mathrm{Mel}$ Lab.tet). Two hundred females from each population were crossed to 200 males from a natively uninfected population collected from locations in Cairns prior to wMel releases. This process was repeated for two further generations to produce a similar nuclear background between populations. The backcrossed $w \mathrm{Mel}$ YK and wMel GV populations were at $\mathrm{F}_{5}$ in the laboratory when experiments commenced.

In both experiments, we measured larval development time, adult wing length, female fecundity and egg hatch proportions. Eggs ( $<1$ week old) from each population were hatched in reverse osmosis (RO) water and 100 larvae ( $<1 \mathrm{~d}$ old) were counted into plastic trays filled with $500 \mathrm{~mL}$ RO water (with 6 replicate trays per population in experiment 1 and 12 replicate trays per population in experiment 2). Larvae were fed TetraMin Tropical Fish Food tablets (Tetra, Melle, Germany) ad libitum until pupation. Development time was scored by counting and sexing pupae twice per day. Adults were pooled across replicate trays and released into cages. Wings of adults (20 males and 20 females per population) were dissected and measured for their length (from the alular notch to the wing tip). Females (5-7 d old, sugar-starved for $1 \mathrm{~d}$ ) were blood fed and isolated in $70 \mathrm{~mL}$ specimen cups filled with $15 \mathrm{~mL}$ of larval rearing water, lined with a strip of sandpaper (Norton Master Painters P80; Saint-Gobain Abrasives Pty. Ltd., Thomastown, Victoria, Australia) and covered with a mesh lid. Twenty females were isolated per population in experiment 1 . In experiment 2 , fecundity and egg hatch proportions were tracked across four consecutive gonotrophic cycles by isolating 30 engorged females per population, returning females that laid eggs to cages, then blood feeding and isolating 30 engorged females again every 4-5 d. Eggs were collected 4 days after blood feeding, partially dried, then hatched $3 \mathrm{~d}$ after collection in trays filled with RO water and a few grains of yeast. Fecundity and egg hatch proportions were determined by counting the total number of eggs and the number of hatched eggs (with the egg cap clearly detached) under a dissecting microscope. In experiment 2 , we also measured adult longevity ( 8 replicate cages of 25 males and 25 females per population) by maintaining adults in $3 \mathrm{~L}$ cages with cups of $10 \%$ sucrose and scoring mortality 3 times per week. Quiescent egg viability was measured in experiment 2 by storing eggs on sandpaper strips in a sealed container with a saturated solution of potassium chloride to maintain a constant humidity of $~ 80 \%$. Twelve replicate batches of eggs per population (median 68 eggs) were hatched on week 1 and 2, then every two weeks until week 22. Egg hatch proportions were scored as above for individual females.

\section{Effects of tetracycline treatment on fitness}

In the above experiments we used populations that had been cleared of Wolbachia infections through tetracycline treatment. Despite allowing for multiple generations of recovery, these treatments could potentially disrupt the microbiome (including the mitochondria), leading to fitness differences between lines that are independent of Wolbachia infection. To test whether antibiotic treatment has any effect on fitness, we fed wMel-infected ( $w$ Mel Lab) and natively uninfected ( $F_{29}$ in the laboratory) adults with 2 $\mathrm{mg} / \mathrm{mL}$ tetracycline hydrochloride for $10 \mathrm{~d}$ before blood feeding, then measured fitness in the subsequent generation. We scored larval development time, survival to pupa and sex ratio ( 6 replicate trays per treatment) as well as female and male wing length, female fecundity and egg hatch proportions (20 individuals each). Experiments were performed identically to the phenotypic comparisons above. 


\section{Cytoplasmic incompatibility}

We tested the ability of wMel-infected Ae. aegypti to induce cytoplasmic incompatibility in near-field and laboratory populations. Reciprocal crosses were performed in June 2019 between wMel-infected populations established from Gordonvale at $F_{1}\left(w\right.$ Mel GV $\left.F_{1}\right)$, Yorkeys Knob at $F_{1}\left(w M e l\right.$ YK $\left.F_{1}\right)$ or Cairns at $F_{60+}$ ( $w$ Mel Lab), and a natively uninfected population ( $F_{35}$ in the laboratory). Pupae from each population were sexed and released into separate $3 \mathrm{~L}$ cages to confirm accurate sex sorting. Groups of 40 females and 40 males ( $1 \mathrm{~d}$ old) were then aspirated into cages together and left for $5 \mathrm{~d}$ to mate. Females (starved of sugar for $24 \mathrm{hr}$ ) were blood-fed, isolated for oviposition and scored for fecundity and egg hatch proportions according to the methods described above for the phenotypic comparisons.

\section{Wolbachia and mitochondrial whole genome sequencing}

Previously, we sequenced the wMel and mitochondrial genomes of Ae. aegypti collected up to eight years after field releases [39]. Here, we extend these findings by sequencing the Wolbachia and mitochondrial genomes of $w$ Mel-infected $A e$. aegypti sampled a decade apart from pre-release and post-release populations. The pre-release wMel-infected populations ( $W C 45 F_{9}$ and $F_{10}$ ) were sampled in 2010 and stored at $-20^{\circ} \mathrm{C}$, while three $w$ Mel-infected populations were sampled in 2020: $w$ Mel GV $F_{2}$ collected from Gordonvale in 2020, wMel YK F $F_{2}$ collected from Yorkeys Knob in 2020, and wMel Lab, collected from Cairns in 2014 and maintained under laboratory conditions until sampling. Genomic DNA was extracted from pooled samples containing five adult females. Sequencing libraries were then prepared as described previously [44].

\section{Reference genome assembly}

Sequencing reads were quality filtered using Trimmomatic [45]. The samples were trimmed in pairedend mode with the following parameter settings: leading =20; trailing = 20; slidingwindow $=4: 20$; minlen =70; adapter sequences were removed using the ILLUMINACLIP option, with maximum seed mismatches $=2$ and the palindrome clip threshold $=30$. Reads were aligned to a $w \mathrm{Mel}$ reference genome (GenBank accession: NC_002978.6) and an Ae. aegypti mitochondrial reference genome (GenBank accession: MH348177.1) with the Burrows-Wheeler Aligner (BWA; [46]) using the bwa mem algorithm and default parameter settings. Quality filtering of alignments and variant calling was performed with SAMtools and BCFtools $[47,48]$. PCR duplicates were excluded from subsequent analyses by soft masking. Reads with a MAPQ score $<25$ were removed from the alignment, except for reads with $M A P Q=0$, which were permitted to allow for mapping to repetitive regions. Genotype likelihoods were calculated using a maximum of 2000 reads per position. For variant calling, ploidy was set to haploid. The variant call output was used to create a consensus nucleotide sequence, wherein genome positions with coverage $<5$ were masked as ' $N$ '. Loci were considered to be polymorphic within a sample if they had coverage $\geq 30$ and two or more alleles with a frequency of $\geq 25 \%$. Any such loci occurring within the $16 \mathrm{~S}$ rRNA or $23 \mathrm{~S}$ rRNA genes were excluded from analysis, as we observed a relatively high level of contaminant reads mapping to these regions. Genome sequences were inspected and aligned with Geneious v 9.1 .8 (https://www.geneious.com). 
Kraken2 [49] and the Standard-8 precompiled reference database (https://benlangmead.github.io/awsindexes/k2; downloaded 17/9/21) were used to search for sequence contamination within the Wolbachia genomes. The sequencing reads mapped by bwa to the wMel reference genome were filtered to remove reads matching taxa other than Wolbachia, and genome assemblies were then repeated with the filtered datasets, using the above pipeline. The original pre-filtration genome sequences were edited to correct erroneous positions after comparison with the corresponding postfiltration genome sequences.

\section{Statistical analysis}

Most experimental data (including Wolbachia density, development time, wing length, fecundity and egg hatch) were analyzed with general linear models (GLMs) while adult longevity data were analysed with log-rank tests. All analyses were carried out with IBM SPSS Statistics 26. Data were transformed where appropriate (with all proportional data being logit transformed). The first experiment had two replicate populations and data were initially analysed with replicate population (nested within population origin $x$ Wolbachia infection status) included as a factor. Replicate populations were then pooled for a second analysis due to a lack of significant effect of replicate population $(P>0.1)$ for any trait. Wolbachia infection status and population origin were included as factors. In the second experiment, fecundity and egg hatch proportions were tracked across gonotrophic cycles, so this was also included as a factor. For quiescent egg viability, wMel-infected and uninfected populations were analysed both together and separately at the first ( 1 week) and last time ( 22 weeks) points. Field Wolbachia density data were analysed with release year and suburb (nested within release year) as factors. We performed Bonferroni corrections where multiple traits were evaluated in the same cohort of mosquitoes.

\section{Results}

wMel remains at a high frequency in Cairns following releases

We performed ovitrapping across suburbs in Cairns in 2016 and 2018 to monitor Wolbachia infection frequencies in the Ae. aegypti population. In 2016, all suburbs where wMel releases had taken place had a wMel infection frequency above 0.96 (Figure S1A). Some pre-release suburbs had low infection frequencies (0.05-0.21), indicating spread of wMel to adjacent suburbs (e.g. to Mt Sheridan and Holloways Beach). By 2018, releases had occurred in additional locations (e.g. Trinity Beach and Redlynch) and all suburbs had wMel infection frequencies greater than 0.93 (Figure S1B), except for Redlynch where wMel was not released until 2019. Our data are broadly consistent with Wolbachia infection frequencies from an independent study which also shows that wMel has maintained high frequencies in most release locations [16].

wMel density remains stable across suburbs and laboratory generations 
We measured $w$ Mel density in $4^{\text {th }}$ instar larvae reared in the laboratory from ovitraps collected across suburbs in 2018. We found no clear effect of release year (GLM: $F_{4,269}=2.274, P=0.062$ ) or suburb (nested within release year) $\left(F_{6,269}=2.017, P=0.064\right)$ on Wolbachia density (Figure $\left.1 \mathrm{~A}\right)$. These results suggest that the wMel infection has remained stable after being established in the field for different periods of time (from 1-7 years), with no clear effects of local environmental conditions on wMel in the next generation of mosquitoes.

In June 2019, we measured wMel density in adults from laboratory populations that were established from field collections at different times. We found no significant differences between populations for females (GLM: $F_{4,65}=1.767, P=0.146$, Figure $\left.1 B\right)$ or males $\left(F_{4,70}=1.942, P=0.113\right.$, Figure $\left.1 C\right)$, suggesting that whole-adult wMel density has not changed across different durations of laboratory rearing. 

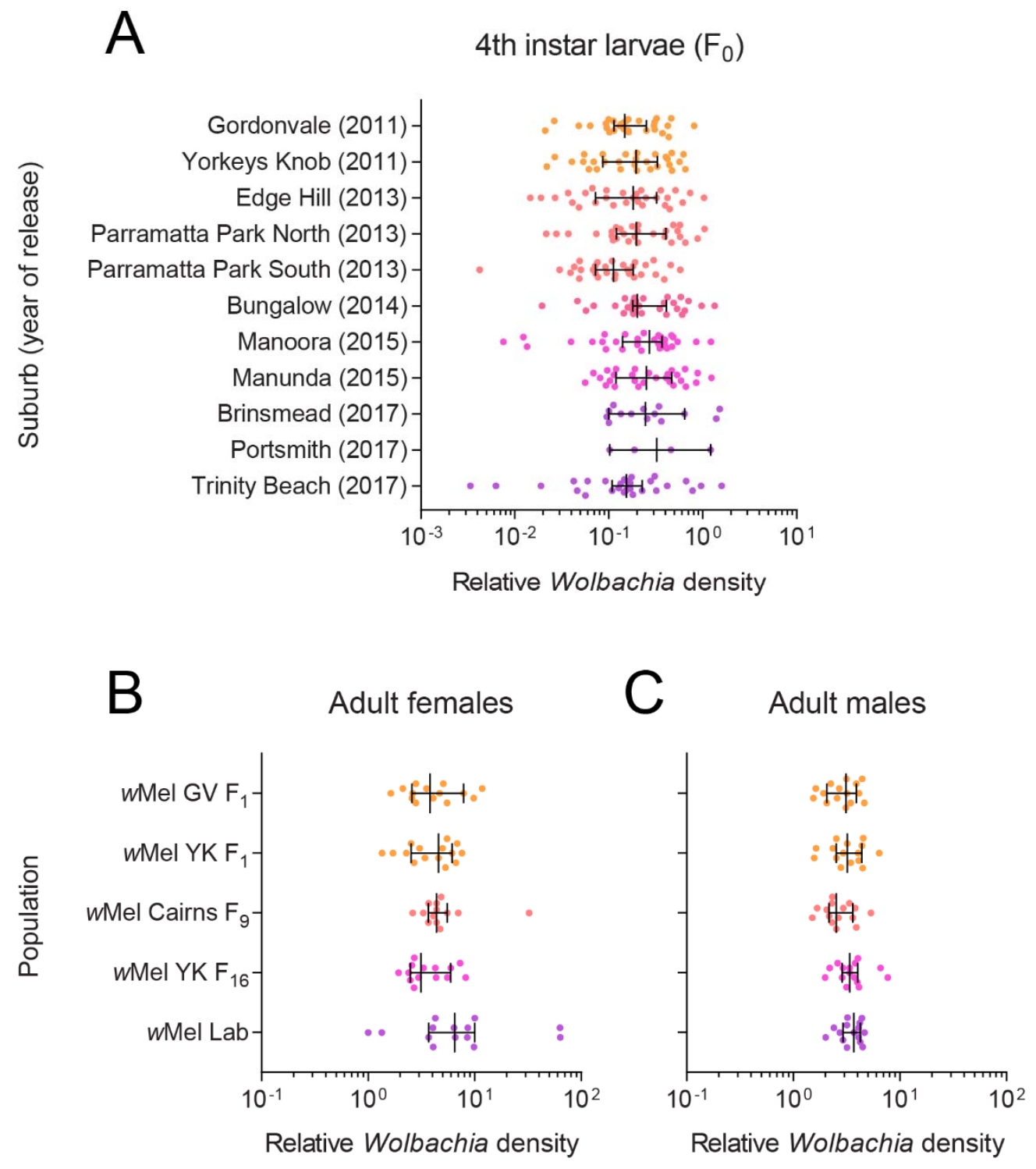

Figure 1. wMel density remains stable across suburbs and laboratory generations. (A) Wolbachia density in $4^{\text {th }}$ instar larvae hatched from ovitraps collected across Cairns suburbs in February-March 2018. The year where releases were undertaken in each suburb is shown in parentheses. (B-C) Wolbachia density in adult (B) females and (C) males in populations that had been reared in the laboratory for different numbers of generations. Dots represent data from three technical replicates of an individual mosquito. Vertical lines and error bars represent medians and $95 \%$ confidence intervals.

\section{Population origin and wMel infection influence mosquito fitness}

In September 2018 we performed phenotypic comparisons between long-term laboratory and near-field populations that were wMel-infected or cleared of wMel through antibiotic treatment (Figure 2). We found significant effects following Bonferroni correction (adjusted $\alpha$ : 0.008 ) of population origin on 
larval development time (females: $F_{1,44}=19.656, P<0.001$, males: $F_{1,44}=18.266, P<0.001$ ) and female fecundity $\left(F_{1,150}=11.640, P=0.001\right)$ but not wing length (females: $F_{1,140}=6.107, P=0.015$, males: $F_{1,141}=$ $0.303, P=0.583$ ) or (logit transformed) egg hatch proportions $\left(F_{1,150}=0.305, P=0.581\right)$. wMel infection had no significant effect on any trait (all $P>0.089$ ) except for male development time $\left(F_{1,44}=9.296, P=\right.$ $0.004)$. For this trait, we also found an interaction between $w$ Mel infection and population origin $\left(F_{1,44}=\right.$ $7.958, \mathrm{P}=0.007$ ), where $w$ Mel infection increased male development time in the lab populations but not the YK (Yorkeys Knob) populations.
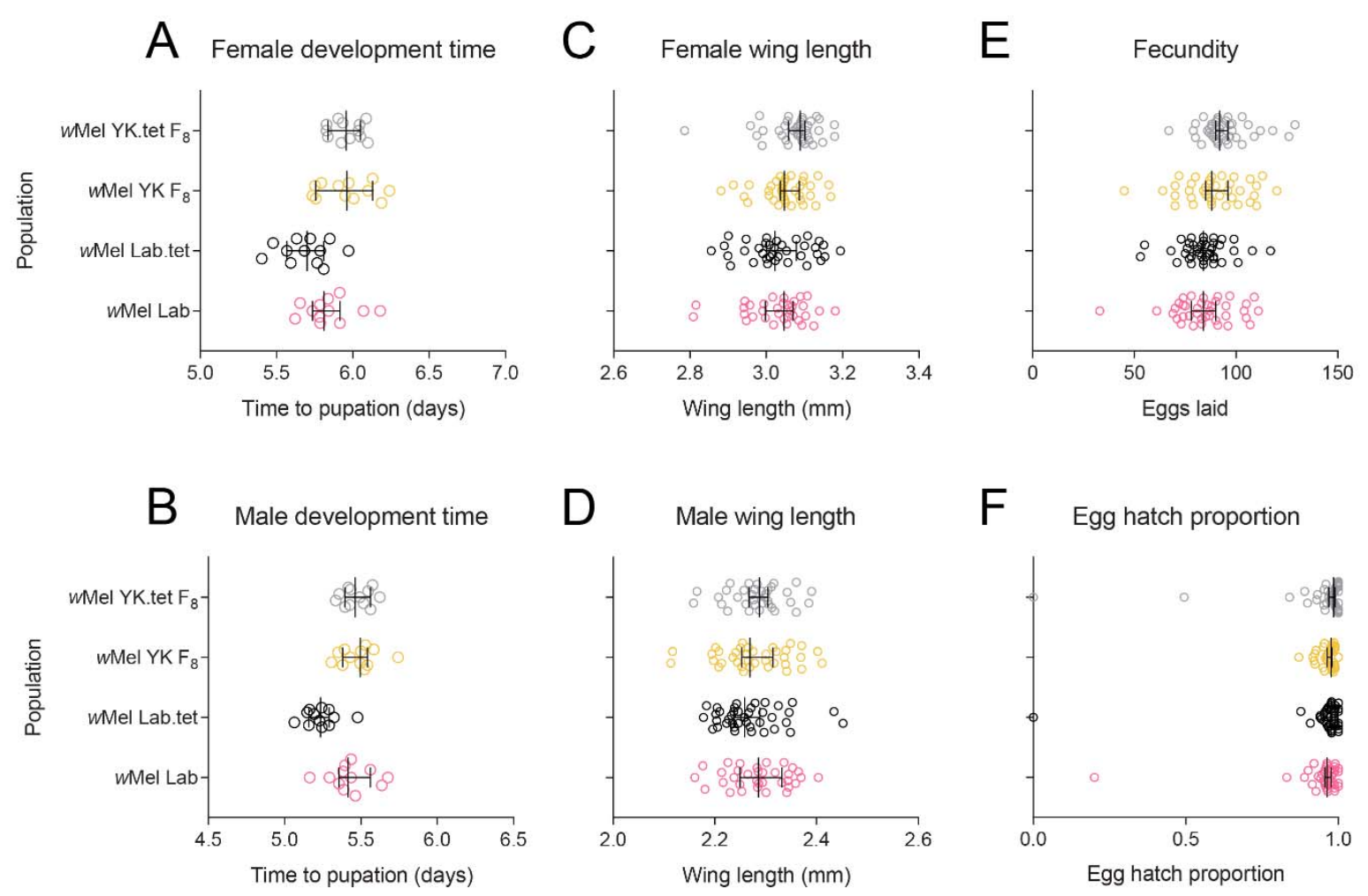

Figure 2. Phenotypic effects of wMel in laboratory and field Aedes aegypti backgrounds. wMelinfected populations were compared to tetracycline-cured counterparts to control for genetic background between infected and uninfected mosquitoes. Populations were measured for $(A)$ female and (B) male development time, (C) female and (D) male wing length, $(E)$ fecundity and (F) egg hatch. Data from two replicate populations were pooled for visualization. Dots represent data from replicate trays (A-B) or individual mosquitoes (C-F). Vertical lines and error bars represent medians and $95 \%$ confidence intervals.

\section{No clear effect of wMel origin in a common host background}

In the first experiment, we found that effects on fitness were driven mainly by population origin. Due to potential interactions between Wolbachia infection and background in the first experiment, we performed a second set of experiments in August 2020 to evaluate the effects of lab and field-derived wMel infections in a common host background. We found no significant differences following 
Bonferroni correction (adjusted $\alpha$ : 0.006) between populations for most traits tested, including development time (GLM: females: $F_{3,43}=2.841, P=0.049$, males: $F_{3,43}=3.558, P=0.022$ ), female wing length $\left(F_{3,74}=1.826, P=0.150\right)$, fecundity $\left(F_{3,433}=1.950, P=0.121\right)$ and adult longevity (Log-rank: females: $\chi^{2}=5.700, P=0.127$, males: $\chi^{2}=3.428, P=0.330$, Figure 3 ). Population effects were observed for male wing length (GLM: $\left.F_{3,73}=5.293, P=0.002\right)$, with $w$ Mel YK males having larger wings (Figure $3 E$ ), and (logit transformed) hatch proportion $\left(F_{3,433}=5.969, P=0.001\right)$, with wMel GV having reduced egg hatch proportions compared to the other populations (Figure 3F). We also found a significant effect of gonotrophic cycle on fecundity $\left(\mathrm{F}_{3,433}=5.808, \mathrm{P}=0.001\right)$, where fecundity was lower in the first cycle (Figure $3 \mathrm{C}$ ). In the quiescent egg viability experiment, we found no significant effect of population on (logit transformed) hatch proportion after 1 week (GLM: $F_{4,55}=1.110, P=0.361$ ). Egg viability declined more rapidly for wMel-infected populations (Figure 3I), with substantial differences between populations by week $22\left(\mathrm{~F}_{4,47}=35.563, \mathrm{P}<0.001\right)$. However, there were no significant differences among $w$ Mel-infected populations at the same time point $\left(F_{2,27}=1.156, P=0.330\right)$. Overall, our results demonstrate few consistent and strong effects of wMel origin on mosquito fitness.
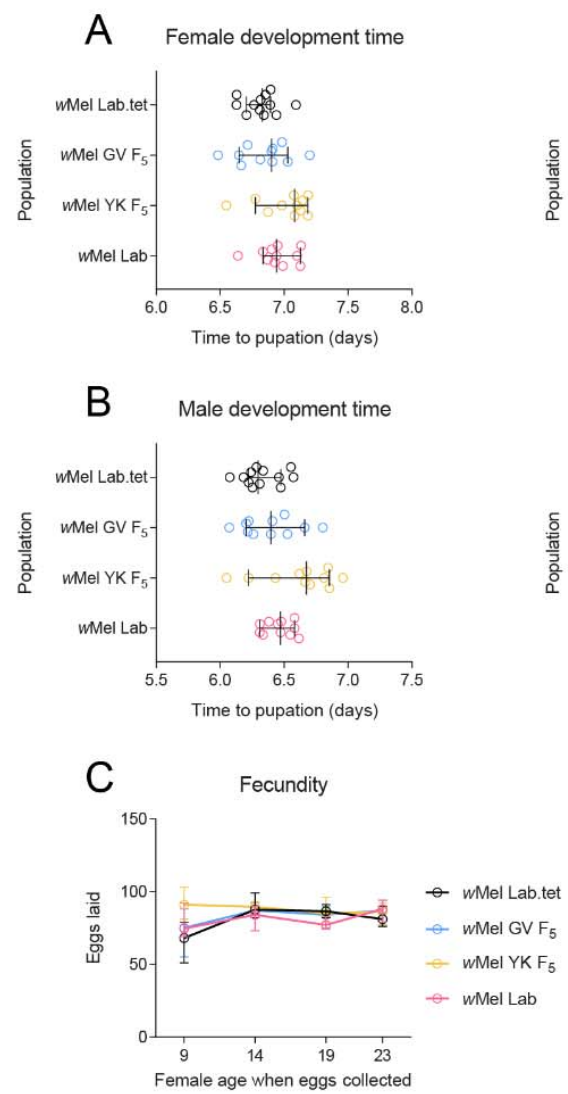
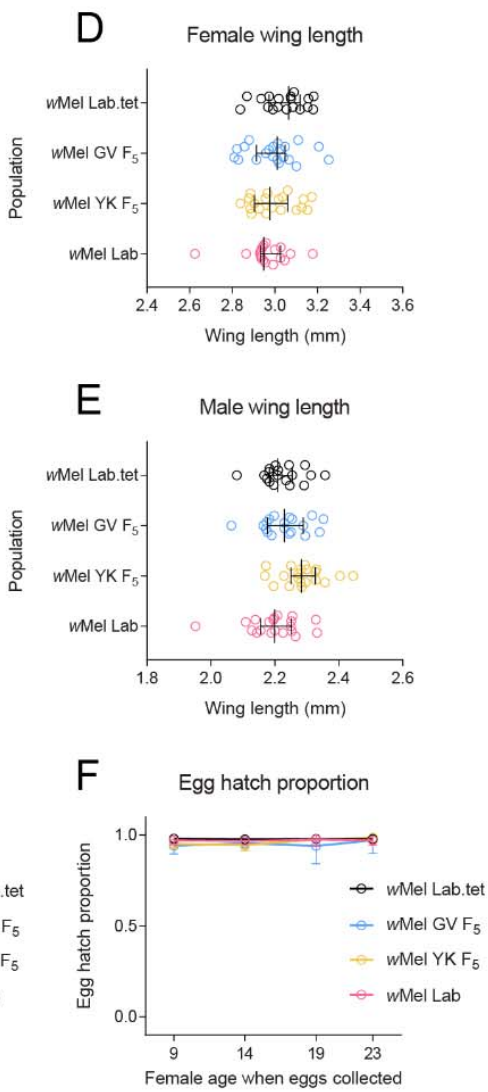
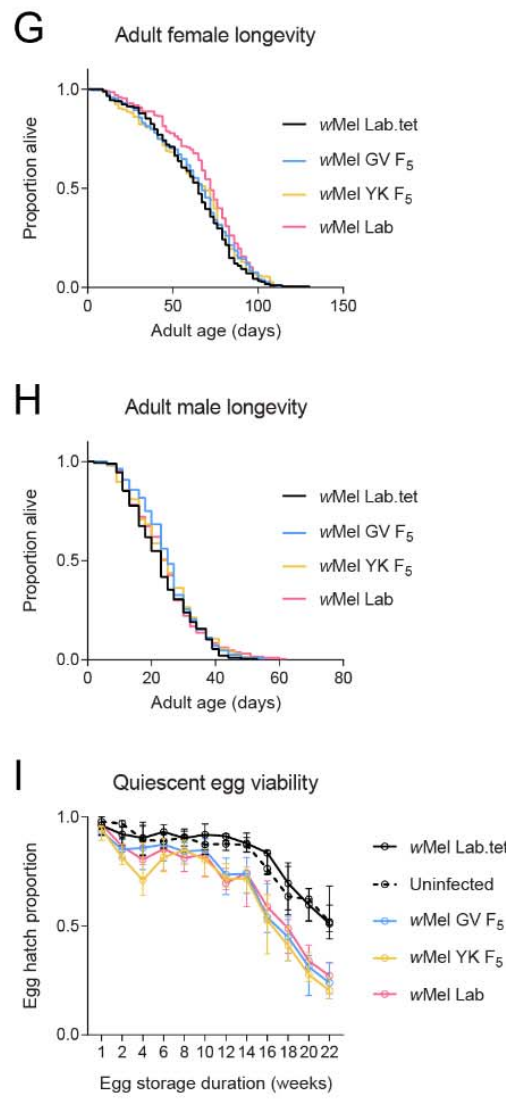

Figure 3. Phenotypic effects of $w$ Mel from lab and field origins in a common Aedes aegypti background. wMel-infections originating from Gordonvale (GV), Yorkeys Knob (YK) or the laboratory were introduced to a common background through backcrossing. Populations were measured for $(A)$ female and (B) male development time, (C) fecundity, (D) female and (E) male wing length, (F) egg hatch, $(\mathrm{G})$ female and $(\mathrm{H})$ male adult longevity and (I) quiescent egg viability. Dots represent data from 
replicate trays (A-B) or individual mosquitoes (D-E). Lines and error bars represent medians and 95\% confidence intervals in all panels.

\section{Limited effects of antibiotic treatment on fitness}

To test whether tetracycline treatment could influence mosquito fitness, we performed phenotypic comparisons of wMel-infected and uninfected mosquitoes following parental tetracycline treatment (Figure 4). We found no significant effect of tetracycline treatment on any trait (all $P>0.007$, adjusted $\alpha$ : 0.006) except for (logit transformed) egg hatch proportion where we found an interaction between treatment and Wolbachia infection type $\left(F_{1,70}=9.261, P=0.003\right)$. Decreased egg hatch due to tetracycline treatment was apparent only for the wMel-infected population, likely due to incomplete curing resulting in partial self-incompatibility. In this experiment, we also found no significant effect of Wolbachia infection type for any trait (all $P>0.017$, adjusted $\alpha: 0.006$ ) except for male wing length, where $w$ Mel-infected males had larger wings $\left(F_{1,69}=12.387, P=0.001\right)$. These results provide confidence that observed effects in comparisons between Wolbachia-infected and cured mosquitoes are due to removal of the Wolbachia infection and not off-target tetracycline-related effects on the gut microbiome or mitochondria.

A
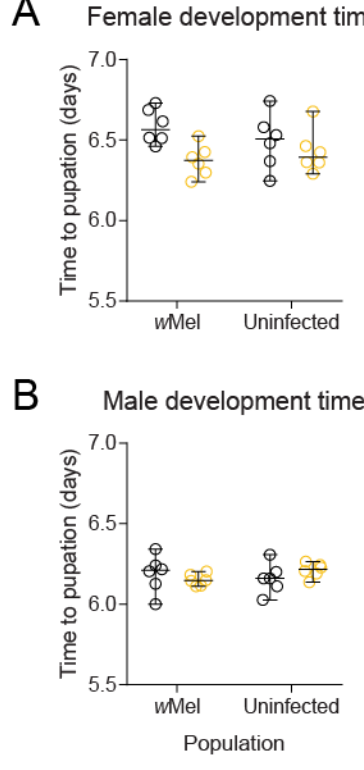
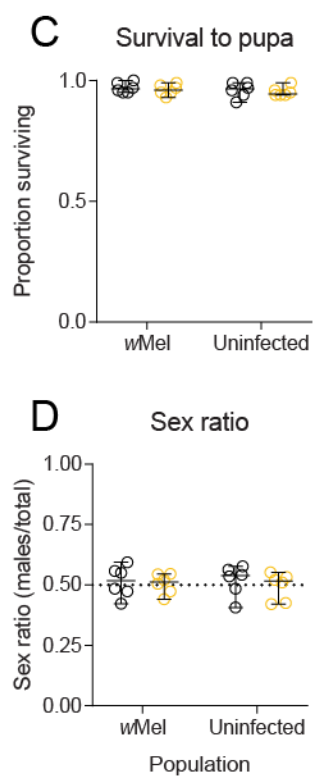
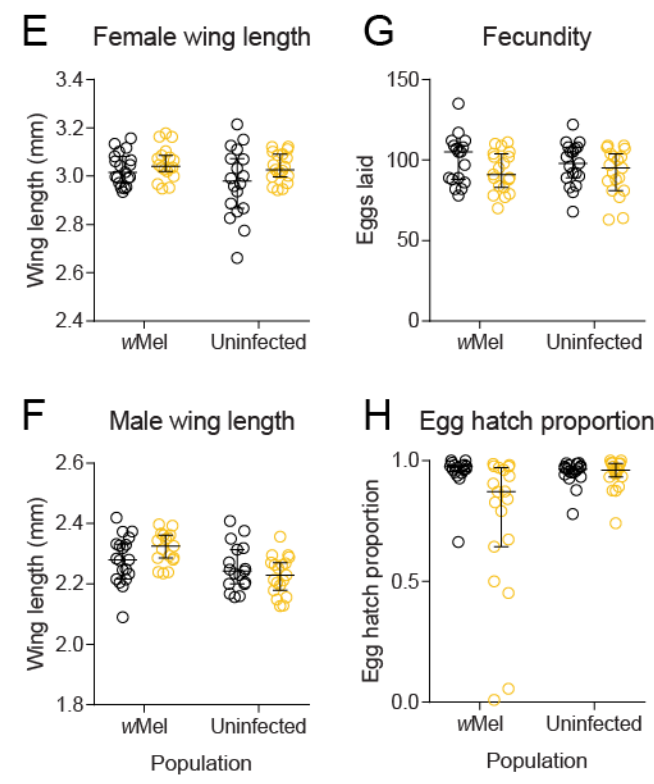

○ Untreated

Tetracycline-treated

Figure 4. Phenotypic effects of parental tetracycline treatment in Aedes aegypti. wMel-infected mosquitoes or uninfected mosquitoes were left untreated or fed $2 \mathrm{mg} / \mathrm{mL}$ tetracycline. Their offspring were measured for (A) female and (B) male development time, (C) survival to pupa, (D) pupal sex ratio, $(E)$ female and $(F)$ male wing length, $(G)$ fecundity and $(H)$ egg hatch. Dots represent data from replicate trays (A-D) or individual mosquitoes $(E-H)$. Lines and error bars represent medians and $95 \%$ confidence intervals in all panels. 
wMel induces complete cytoplasmic incompatibility after eight years in the field

In June 2019, we tested the ability of wMel-infected males from different origins (Yorkeys Knob, Gordonvale and laboratory) to induce cytoplasmic incompatibility with uninfected females. Males from all wMel-infected populations induced complete cytoplasmic incompatibility, with no eggs hatching in crosses with uninfected females (Table 1). All other crosses yielded egg hatch proportions above $90 \%$ (Table 1), consistent with data from the previous experiments showing limited effects of wMel infection on egg hatch. These results show that $w$ Mel has retained complete cytoplasmic incompatibility after $>8$ years in the field and one generation of laboratory rearing.

Table 1. Reciprocal crosses between wMel-infected Aedes aegypti from laboratory or field origins and uninfected $\boldsymbol{A e}$. aegypti. Crosses with "-" were not tested.

\begin{tabular}{|c|c|c|c|c|c|}
\hline & & \multicolumn{4}{|c|}{$\begin{array}{l}\text { Median egg hatch proportion (lower, upper 95\% confidence } \\
\text { interval) }\end{array}$} \\
\hline & & \multicolumn{4}{|c|}{ Female } \\
\hline & & Uninfected & wMel Lab & $w$ Mel YK F & wMel GV F \\
\hline \multirow[t]{4}{*}{ Male } & Uninfected & $\begin{array}{l}0.946(0.922 \\
0.971)\end{array}$ & $\begin{array}{l}0.960(0.915 \\
0.981)\end{array}$ & $\begin{array}{l}0.958(0.800, \\
0.973)\end{array}$ & $\begin{array}{l}0.974(0.964, \\
0.981)\end{array}$ \\
\hline & wMel Lab & $0(0,0)$ & $\begin{array}{l}0.959(0.949, \\
0.978)\end{array}$ & - & - \\
\hline & $w \mathrm{Mel} \mathrm{YK} \mathrm{F}_{1}$ & $0(0,0)$ & - & $\begin{array}{l}0.973(0.924, \\
0.991)\end{array}$ & - \\
\hline & wMel GV F & $0(0,0)$ & - & - & $\begin{array}{l}0.974(0.958, \\
0.991)\end{array}$ \\
\hline
\end{tabular}

Limited changes in wMel and mitochondrial genomes across a decade in a novel Aedes aegypti host

Previous wMel genome comparisons suggest limited changes in the Wolbachia genome have occurred since field releases $[39,40]$. We extend this timeframe by including pre-release material from 2010 and field collections from Gordonvale and Yorkeys Knob in 2020. We also include a dataset from a previous whole genome sequencing study of individuals collected from Gordonvale in 2013 and 2018 and Yorkey's Knob in 2018 [38]. Little evidence of change was observed among the analysed wMel genomes, for which we achieved $\geq 99.99 \%$ coverage. Within populations, each of which was represented by a sample of pooled individuals, seven loci were found to have one or more alternate alleles present at a frequency greater than our threshold value of $25 \%$ in at least one sample, all of which were SNPs or small indels. At all other positions within the genome, the $w C 45 \mathrm{~F}_{10}$ (pre-release) consensus sequence was identical to that of the Gordonvale and Yorkeys Knob field collections from 2011, 2018 and 2020, and the wMel lab population (see also Huang et al. [39] and Dainty et al. [40]). Relative to these genomes, one single nucleotide deletion was observed in the $w \mathrm{C}_{45} \mathrm{~F}_{9}$ pre-release genome sequence and seven single nucleotide indels were detected in the 2013 Gordonvale field collection genome sequence (Table S1). It is probable that these eight indels represent sequencing artifacts, as they involve non-parsimonious changes in populations sampled over multiple time points, were often associated with a marked localized decrease in read depth in the variant samples and mostly represent frameshift mutations. 
Of the seven loci that displayed within-sample polymorphism, two were within non-coding regions; three were within three different copies of the group II intron-encoded protein gene LtrA, each at the same position within the gene and corresponding to an Asp > Asn substitution; one was within a gene encoding a hypothetical protein and corresponds to a silent change; and one was within a tRNA-Arg gene (Table S2). It is possible that the apparent polymorphism within the LtrA genes is due to sequence variation between gene copies, rather than the presence of alternate alleles. Multiple alleles were observed in all samples for these three loci and for the polymorphic locus within the tRNA-Arg gene. At the other three loci, allele frequencies were more variable, with only some samples displaying polymorphism, most of which were from the field populations at Gordonvale and Yorkeys Knob. Two of these loci, at positions 587,862 and 1,174,712 relative to the wMel reference genome, were previously noted by Huang et al. [39] and Dainty et al. [40]. No clear pattern was observed across different populations or time points. While it is possible that this variation is attributable to underlying fluctuation in allele frequencies within populations, or methodological differences between studies, it is more likely to be due to stochastic sampling effects, given the relatively small number of individuals pooled in some samples.

Similar to the wMel genomes, we detected very few changes between the mitochondrial genomes sequenced in this study, for which we obtained $\geq 99.31 \%$ coverage. The genomes shared $100 \%$ sequence identity across all gene-encoding regions. Nucleotide differences were observed among the consensus sequences at 24 positions within an approximately $1.8 \mathrm{~Kb}$ region of the genome that does not contain any predicted gene sequences, is very AT rich and displayed greatly reduced read mapping depths (Table S1). It is therefore likely that many or all of these differences represent sequencing errors. A further 16 positions within this region were found to show within-sample polymorphism, with alternate alleles present at a frequency greater than the threshold value in at least one sample (Table S2). The lack of variation observed among the wMel genomes and among the mitochondrial genomes is consistent with a high fidelity of maternal co-transmission of both Wolbachia and mitochondria.

\section{Discussion}

In this study, we provide a comprehensive update on the genomic and phenotypic stability of the wMel Wolbachia infection, up to ten years after being released in Ae. aegypti populations. We observed few changes in the wMel genome since before releases began, with little divergence between different locations despite nearly a decade of separation. Furthermore, wMel retains complete cytoplasmic incompatibility, a stable within-host density, and limited host fitness costs. Maintenance of these phenotypic effects along with virus blocking $[21,36]$ and maternal transmission [15] is crucial for the ongoing persistence of Wolbachia and suppression of arbovirus transmission. Our results add to a growing body of evidence (e.g. $[15,16,39]$ ) supporting the notion that wMel will continue to remain an effective tool for dengue control for many years.

Our results suggest that any shifts in the host phenotypic effects of wMel are likely to reflect evolutionary changes within mosquito populations rather than the Wolbachia genome. This hypothesis is consistent with crossing and selection experiments showing that Wolbachia phenotypic effects depend on nuclear background $[13,50]$. It is also consistent with genomic data from this and previous 
studies showing the long-term stability of Wolbachia genomes following transinfection [33, 34, 39, 40]. Our fitness experiments do not provide evidence for evolutionary changes in response to Wolbachia infection, with similar effects observed in mosquito populations that had been infected with wMel for many years (experiment 1 ) and in populations where wMel was recently introgressed (experiment 2). Comparisons of mosquito genomes prior to wMel release in Gordonvale and seven years post-release point to limited changes [38], but further work with replicated populations is required to confirm any changes due to Wolbachia infection.

Accurate phenotyping of Wolbachia infections requires careful control of the nuclear and mitochondrial background between Wolbachia-infected and uninfected populations. In this study, we used tetracycline curing followed by backcrossing to ensure that Wolbachia-infected and uninfected populations had matched mitochondrial and nuclear genomes. Cross-generational effects of tetracycline treatment on fitness have been speculated [51], with some studies accounting for potential disruptions to the microbiome by rearing treated mosquitoes in water from untreated mosquitoes (e.g. [52]). We found no evidence to suggest that tetracycline treatment influences fitness in the following generation, at least for a concentration commonly used to cure Wolbachia infections in insects [53]. While the effect of tetracycline treatment on the mosquito microbiome (aside from Wolbachia) remains to be tested, repeated backcrossing to a common background should help to minimize any differences between populations.

Our data, spanning up to a decade since the first releases of wMel in Australia, should be informative for Wolbachia release programs that have taken place more recently in other countries. Our results suggest that when the wMel infection is maintained in an Ae. aegypti population, the phenotypic effects associated with $w \mathrm{Mel}$ invasion are likely to persist given that the infection remains at a high density. However, more work is required to understand the extent of genetic changes in mosquito populations in response to Wolbachia releases. Finally, while wMel has retained complete cytoplasmic incompatibility and maternal transmission under laboratory conditions, these parameters are affected by environmental conditions [10] and require further evaluation under field conditions. The persistence of a high incidence of wMel in natural populations may be constrained by environmental conditions including high temperatures in some locations [25]. Ongoing monitoring remains important to identify any changes in Wolbachia infection frequency and inform the need for supplementary releases including those with different strains.

\section{Supplementary information}


(A) September-October 2016

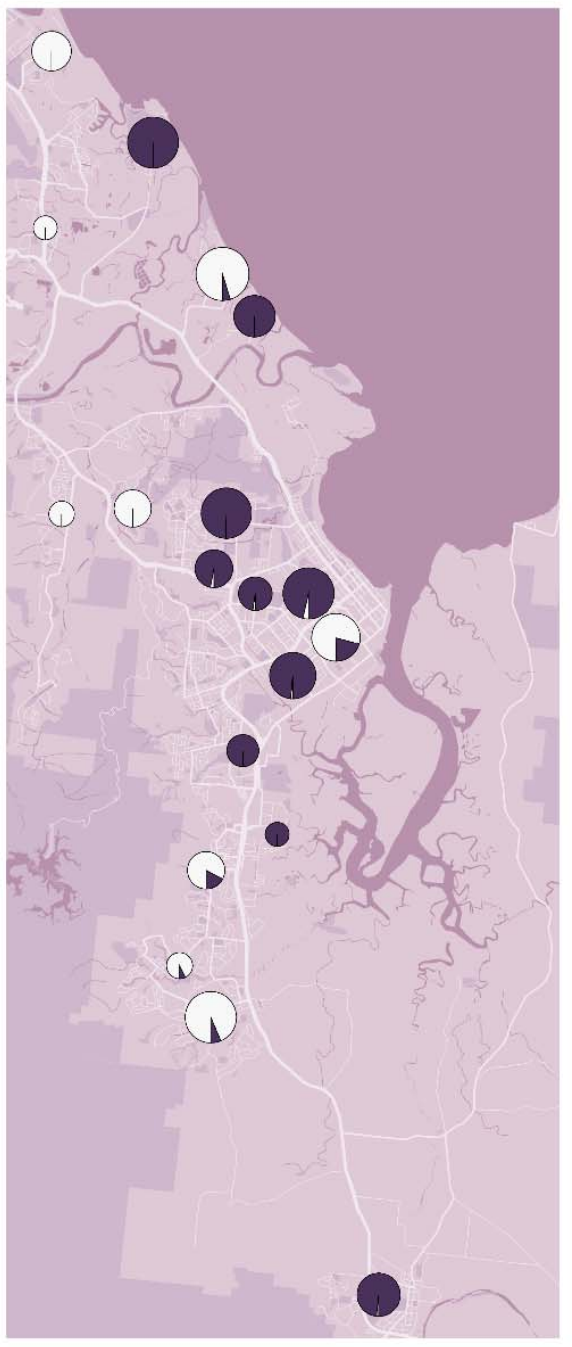

(B) February-March 2018

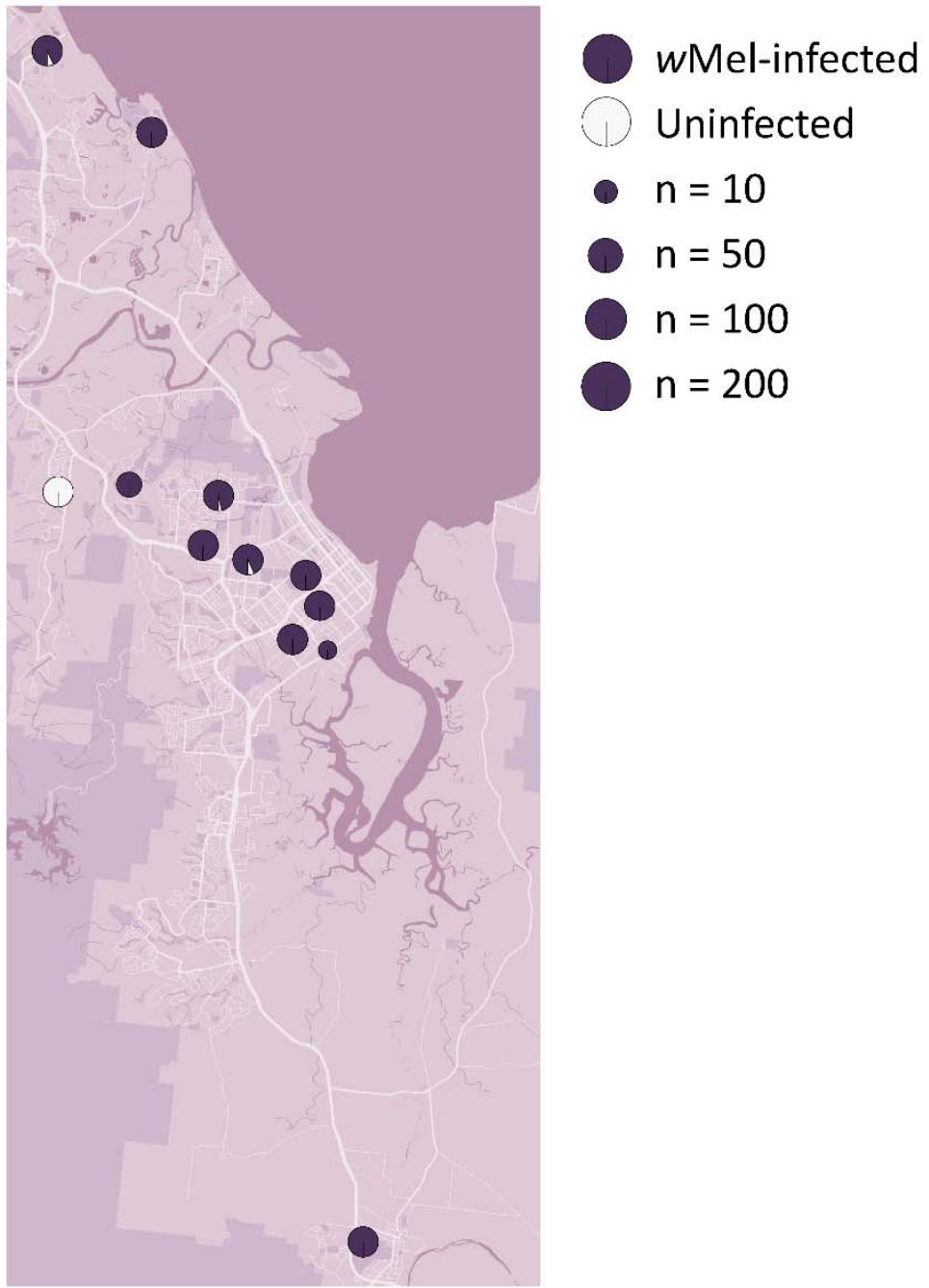

Figure S1. wMel Wolbachia infection frequencies in Cairns in (A) 2016 and (B) 2018 sampled through ovitrapping.

Table S1. Observed differences in Wolbachia and mitochondrial genomes. Positions are shown relative to the GenBank reference sequences used for read mapping. Some or all of the differences are likely to be sequencing artifacts (see main text). For comparison, the results of Huang et al. [39] and Dainty et al. [40] are also shown. ND = not determined.

Table S2: Genome positions found to show polymorphism within populations. In our study, loci were considered to be polymorphic within a sample if they had coverage $\geq 30$ and two or more alleles with a frequency of $\geq 25 \%$. Any such loci occurring within the 165 rRNA or 235 rRNA genes were excluded from analysis, as we observed a relatively high level of contaminant reads mapping to these regions. Positions are shown relative to the GenBank reference sequences used for read mapping. For comparison, the results of Huang et al. [39] and Dainty et al. [40] are also shown. For Dainty et al. [40], multiple samples 
per population were analysed, with each sample containing a single individual - allele frequencies are reported as number individuals/total individuals per population. NR = not reported; not scored as polymorphic by the authors of the original study; in most cases these values are likely to be at or close to $100 \%$.

\section{Acknowledgements}

We thank Safi Soleimannejad and Kelly Richardson for technical assistance.

\section{Data availability}

wMel genome sequences will be deposited in Genbank following submission.

\section{References}

1. Ross PA, Turelli M, Hoffmann AA. Evolutionary ecology of Wolbachia releases for disease control. Annu Rev Genet. 2019;53(1):93-116. doi: 10.1146/annurev-genet-112618-043609.

2. Wang G-H, Gamez S, Raban RR, Marshall JM, Alphey L, Li M, et al. Combating mosquito-borne diseases using genetic control technologies. Nature communications. 2021;12(1):4388. doi: 10.1038/s41467-021-24654-z.

3. Caragata EP, Dutra HLC, Sucupira PHF, Ferreira AGA, Moreira LA. Wolbachia as translational science: controlling mosquito-borne pathogens. Trends Parasitol. 2021. doi:

https://doi.org/10.1016/i.pt.2021.06.007.

4. Moreira LA, Iturbe-Ormaetxe I, Jeffery JA, Lu G, Pyke AT, Hedges LM, et al. A Wolbachia symbiont in Aedes aegypti limits infection with dengue, Chikungunya, and Plasmodium. Cell. 2009;139(7):1268-78. doi: 10.1016/j.cell.2009.11.042. PubMed PMID: 20064373.

5. van den Hurk AF, Hall-Mendelin S, Pyke AT, Frentiu FD, McElroy K, Day A, et al. Impact of Wolbachia on infection with chikungunya and yellow fever viruses in the mosquito vector Aedes aegypti. PLoS neglected tropical diseases. 2012;6(11):e1892. doi: 10.1371/journal.pntd.0001892. PubMed PMID: 23133693; PubMed Central PMCID: PMC3486898.

6. Dutra HL, Rocha MN, Dias FB, Mansur SB, Caragata EP, Moreira LA. Wolbachia blocks currently circulating Zika virus isolates in Brazilian Aedes aegypti mosquitoes. Cell host \& microbe. 2016;19(6):771-4. doi: 10.1016/j.chom.2016.04.021. PubMed PMID: 27156023.

7. Nazni WA, Hoffmann AA, NoorAfizah A, Cheong YL, Mancini MV, Golding N, et al. Establishment of Wolbachia strain wAlbB in Malaysian populations of Aedes aegypti for dengue control. Current biology : CB. 2019;29(24):4241-8 e5. Epub 2019/11/26. doi: 10.1016/j.cub.2019.11.007. PubMed PMID: 31761702; PubMed Central PMCID: PMCPMC6926472.

8. Utarini A, Indriani C, Ahmad RA, Tantowijoyo W, Arguni E, Ansari MR, et al. Efficacy of Wolbachia-infected mosquito deployments for the control of dengue. N Engl J Med. 2021;384(23):217786. doi: 10.1056/NEJMoa2030243. PubMed PMID: 34107180.

9. Schmidt TL, Filipovic I, Hoffmann AA, Rasic G. Fine-scale landscape genomics helps explain the slow spatial spread of Wolbachia through the Aedes aegypti population in Cairns, Australia. Heredity. 2018;120:386-95. Epub 2018/01/24. doi: 10.1038/s41437-017-0039-9. PubMed PMID: 29358725.

10. Ross PA, Wiwatanaratanabutr I, Axford JK, White VL, Endersby-Harshman NM, Hoffmann AA. Wolbachia infections in Aedes aegypti differ markedly in their response to cyclical heat stress. PLoS pathogens. 2017;13(1):e1006006. doi: 10.1371/journal.ppat.1006006. 
11. Ross PA, Ritchie SA, Axford JK, Hoffmann AA. Loss of cytoplasmic incompatibility in Wolbachiainfected Aedes aegypti under field conditions. PLoS neglected tropical diseases. 2019;13(4):e0007357. doi: 10.1371/journal.pntd.0007357.

12. Lau M-J, Ross PA, Hoffmann AA. Infertility and fecundity loss of Wolbachia-infected Aedes aegypti hatched from quiescent eggs is expected to alter invasion dynamics. PLoS neglected tropical diseases. 2021;15(2):e0009179. doi: https://doi.org/10.1101/2020.11.25.397240.

13. Ritchie SA, Townsend M, Paton CJ, Callahan AG, Hoffmann AA. Application of wMelPop Wolbachia strain to crash local populations of Aedes aegypti. PLoS neglected tropical diseases. 2015;9(7):e0003930. doi: 10.1371/journal.pntd.0003930. PubMed PMID: 26204449; PubMed Central PMCID: PMC4512704.

14. Hoffmann AA, Montgomery BL, Popovici J, Iturbe-Ormaetxe I, Johnson PH, Muzzi F, et al. Successful establishment of Wolbachia in Aedes populations to suppress dengue transmission. Nature. 2011;476(7361):454-7. doi: 10.1038/nature10356. PubMed PMID: 21866160.

15. Hoffmann AA, Iturbe-Ormaetxe I, Callahan AG, Phillips BL, Billington K, Axford JK, et al. Stability of the wMel Wolbachia infection following invasion into Aedes aegypti populations. PLoS neglected tropical diseases. 2014;8(9):e3115. doi: 10.1371/journal.pntd.0003115. PubMed PMID: 25211492.

16. Ryan PA, Turley AP, Wilson G, Hurst TP, Retzki K, Brown-Kenyon J, et al. Establishment of wMel Wolbachia in Aedes aegypti mosquitoes and reduction of local dengue transmission in Cairns and surrounding locations in northern Queensland, Australia. Gates Open Res. 2019;3. doi: 10.12688/gatesopenres.13061.1.

17. O'Neill SL, Ryan PA, Turley AP, Wilson G, Retzki K, Iturbe-Ormaetxe I, et al. Scaled deployment of Wolbachia to protect the community from dengue and other Aedes transmitted arboviruses. Gates Open Res. 2018;2.

18. Tantowijoyo W, Andari B, Arguni E, Budiwati N, Nurhayati I, Fitriana I, et al. Stable establishment of wMel Wolbachia in Aedes aegypti populations in Yogyakarta, Indonesia. PLoS neglected tropical diseases. 2020;14(4):e0008157. Epub 2020/04/18. doi: 10.1371/journal.pntd.0008157. PubMed PMID: 32302295; PubMed Central PMCID: PMCPMC7190183.

19. Garcia GdA, Sylvestre G, Aguiar R, da Costa GB, Martins AJ, Lima JBP, et al. Matching the genetics of released and local Aedes aegypti populations is critical to assure Wolbachia invasion. PLoS neglected tropical diseases. 2019;13(1):e0007023. doi: 10.1371/journal.pntd.0007023.

20. Gesto JSM, Pinto S, Dias FBS, Peixoto J, Costa G, Kutcher S, et al. Large-scale deployment and establishment of Wolbachia into the Aedes aegypti population in Rio de Janeiro, Brazil. bioRxiv. 2021:2021.04.29.441982. doi: 10.1101/2021.04.29.441982.

21. Gesto JSM, Ribeiro GS, Rocha MN, Dias FBS, Peixoto J, Carvalho FD, et al. Reduced competence to arboviruses following the sustainable invasion of Wolbachia into native Aedes aegypti from Southeastern Brazil. Scientific reports. 2021;11(1):1-14.

22. Pinto SB, Riback TIS, Sylvestre G, Costa G, Peixoto J, Dias FBS, et al. Effectiveness of Wolbachiainfected mosquito deployments in reducing the incidence of dengue and other Aedes-borne diseases in Niterói, Brazil: A quasi-experimental study. PLoS neglected tropical diseases. 2021;15(7):e0009556. doi: 10.1371/journal.pntd.0009556.

23. Indriani C, Tantowijoyo W, Rancès E, Andari B, Prabowo E, Yusdi D, et al. Reduced dengue incidence following deployments of Wolbachia-infected Aedes aegypti in Yogyakarta, Indonesia: a quasiexperimental trial using controlled interrupted time series analysis. Gates Open Res. 2020;4(50). doi: 10.12688/gatesopenres.13122.1.

24. Ross PA, Axford JK, Yang Q, Staunton KM, Ritchie SA, Richardson KM, et al. Heatwaves cause fluctuations in wMel Wolbachia densities and frequencies in Aedes aegypti. PLoS neglected tropical diseases. 2020;14(1):e0007958. Epub 2020/01/24. doi: 10.1371/journal.pntd.0007958. PubMed PMID: 31971938; PubMed Central PMCID: PMCPMC6977724. 
25. Hien NT, Anh DD, Le NH, Yen NT, Phong TV, Nam VS, et al. Environmental factors influence the local establishment of Wolbachia in Aedes aegypti mosquitoes in two small communities in central Vietnam. Gates Open Res. 2021;5(147):147.

26. Bull JJ, Turelli M. Wolbachia versus dengue: Evolutionary forecasts. Evolution, medicine, and public health. 2013;2013(1):197-207. doi: 10.1093/emph/eot018. PubMed PMID: 24481199; PubMed Central PMCID: PMC3847891.

27. Edenborough KM, Flores HA, Simmons CP, Fraser JE, Pierson TC. Using Wolbachia to eliminate dengue: Will the virus fight back? Journal of virology. 2021;95(13):e02203-20. doi: doi:10.1128/JVI.02203-20.

28. Ritchie SA, van den Hurk AF, Smout MJ, Staunton KM, Hoffmann AA. Mission accomplished? We need a guide to the 'post release' world of Wolbachia for Aedes-borne disease control. Trends Parasitol. 2018. doi: 10.1016/j.pt.2017.11.011.

29. Weeks AR, Turelli M, Harcombe WR, Reynolds KT, Hoffmann AA. From parasite to mutualist: rapid evolution of Wolbachia in natural populations of Drosophila. PLoS biology. 2007;5(5):e114.

30. McGraw EA, Merritt DJ, Droller JN, O'Neill SL. Wolbachia density and virulence attenuation after transfer into a novel host. Proc Natl Acad Sci USA. 2002;99(5):2918-23. doi: 10.1073/pnas.052466499. PubMed PMID: 11880639; PubMed Central PMCID: PMC122448.

31. Carrington LB, Hoffmann AA, Weeks AR. Monitoring long-term evolutionary changes following Wolbachia introduction into a novel host: the Wolbachia popcorn infection in Drosophila simulans. Proc R Soc B Biol Sci. 2010;277(1690):2059-68. doi: 10.1098/rspb.2010.0166. PubMed PMID: 20219740; PubMed Central PMCID: PMC2880104.

32. Carrington LB, Leslie J, Weeks AR, Hoffmann AA. The popcorn Wolbachia infection of Drosophila melanogaster: can selection alter Wolbachia longevity effects? Evolution; international journal of organic evolution. 2009;63(10):2648-57. doi: 10.1111/j.1558-5646.2009.00745.x. PubMed PMID: 19500146.

33. Morrow JL, Schneider DI, Klasson L, Janitz C, Miller WJ, Riegler M. Parallel Sequencing of Wolbachia wCer2 from donor and novel hosts reveals multiple incompatibility factors and genome stability after host transfers. Genome biology and evolution. 2020;12(5):720-35. Epub 2020/03/13. doi: 10.1093/gbe/evaa050. PubMed PMID: 32163151; PubMed Central PMCID: PMCPMC7259677.

34. Woolfit M, Iturbe-Ormaetxe I, Brownlie JC, Walker T, Riegler M, Seleznev A, et al. Genomic evolution of the pathogenic Wolbachia strain, wMelPop. Genome biology and evolution.

2013;5(11):2189-204. doi: 10.1093/gbe/evt169. PubMed PMID: 24190075; PubMed Central PMCID: PMC3845649.

35. Ross PA, Axford JK, Callahan AG, Richardson KM, Hoffmann AA. Persistent deleterious effects of a deleterious Wolbachia infection. PLoS neglected tropical diseases. 2020;14(4):e0008204. Epub 2020/04/04. doi: 10.1371/journal.pntd.0008204. PubMed PMID: 32243448; PubMed Central PMCID: PMCPMC7159649.

36. Frentiu FD, Zakir T, Walker T, Popovici J, Pyke AT, van den Hurk A, et al. Limited dengue virus replication in field-collected Aedes aegypti mosquitoes infected with Wolbachia. PLoS neglected tropical diseases. 2014;8(2):e2688. doi: 10.1371/journal.pntd.0002688. PubMed PMID: 24587459; PubMed Central PMCID: PMC3930499.

37. Ross PA, Hoffmann AA. Continued susceptibility of the wMel Wolbachia infection in Aedes aegypti to heat stress following field deployment and selection. Insects. 2018;9(3). Epub 2018/07/04. doi: 10.3390/insects9030078. PubMed PMID: 29966368; PubMed Central PMCID: PMCPMC6165456. 38. Lau M-J, Schmidt T, Yang Q, Chung J, Sankey L, Ross PA, et al. Genetic stability of Aedes aegypti populations following invasion by wMel Wolbachia. bioRxiv. 2021:2021.06.03.446908. doi: 10.1101/2021.06.03.446908. 
39. Huang B, Yang Q, Hoffmann AA, Ritchie SA, van den Hurk AF, Warrilow D. Wolbachia genome stability and mtDNA variants in Aedes aegypti field populations eight years after release. iScience. 2020;23(10):101572. Epub 2020/10/22. doi: 10.1016/j.isci.2020.101572. PubMed PMID: 33083739; PubMed Central PMCID: PMCPMC7527712.

40. Dainty KR, Hawkey J, Judd LM, Pacidônio EC, Duyvestyn JM, Gonçalves DS, et al. wMel Wolbachia genome remains stable after 7 years in Australian Aedes aegypti field populations. Microb Genom. 2021;7(9):000641.

41. Schmidt TL, Barton NH, Rasic G, Turley AP, Montgomery BL, Iturbe-Ormaetxe I, et al. Local introduction and heterogeneous spatial spread of dengue-suppressing Wolbachia through an urban population of Aedes aegypti. PLoS biology. 2017;15(5):e2001894. doi: 10.1371/journal.pbio.2001894. PubMed PMID: 28557993.

42. Ross PA, Axford JK, Richardson KM, Endersby-Harshman NM, Hoffmann AA. Maintaining Aedes aegypti mosquitoes infected with Wolbachia. Journal of visualized experiments : JoVE. 2017;(126):e56124. doi: 10.3791/56124.

43. Lee SF, White VL, Weeks AR, Hoffmann AA, Endersby NM. High-throughput PCR assays to monitor Wolbachia infection in the dengue mosquito (Aedes aegypti) and Drosophila simulans. Applied and environmental microbiology. 2012;78(13):4740-3. doi: 10.1128/AEM.00069-12. PubMed PMID: 22522691; PubMed Central PMCID: PMC3370494.

44. Ross PA, Gu X, Robinson KL, Yang Q, Cottingham E, Zhang Y, et al. A wAlbB Wolbachia transinfection displays stable phenotypic effects across divergent Aedes aegypti mosquito backgrounds. Applied and environmental microbiology. 2021;87(20):e01264-21.

45. Bolger AM, Lohse M, Usadel B. Trimmomatic: a flexible trimmer for Illumina sequence data. Bioinformatics. 2014;30(15):2114-20.

46. Li H. Aligning sequence reads, clone sequences and assembly contigs with BWA-MEM. arXiv. 2013:1303.3997.

47. Li H, Handsaker B, Wysoker A, Fennell T, Ruan J, Homer N, et al. The sequence alignment/map format and SAMtools. Bioinformatics. 2009;25(16):2078-9.

48. Danecek P, Bonfield JK, Liddle J, Marshall J, Ohan V, Pollard MO, et al. Twelve years of SAMtools and BCFtools. GigaScience. 2021;10(2):giab008.

49. Wood DE, Lu J, Langmead B. Improved metagenomic analysis with Kraken 2. Genome biology. 2019;20(1):1-13.

50. Ford SA, Allen SL, Ohm JR, Sigle LT, Sebastian A, Albert I, et al. Selection on Aedes aegypti alters Wolbachia-mediated dengue virus blocking and fitness. Nat Microbiol. 2019;4(11):1832-9. Epub 2019/08/28. doi: 10.1038/s41564-019-0533-3. PubMed PMID: 31451771; PubMed Central PMCID: PMCPMC6990461.

51. Ballard J, Melvin R. Tetracycline treatment influences mitochondrial metabolism and mtDNA density two generations after treatment in Drosophila. Insect molecular biology. 2007;16(6):799-802. 52. McMeniman CJ, Lane RV, Cass BN, Fong AW, Sidhu M, Wang Y-F, et al. Stable introduction of a life-shortening Wolbachia infection into the mosquito Aedes aegypti. Science. 2009;323(5910):141-4. 53. Li YY, Floate KD, Fields PG, Pang BP. Review of treatment methods to remove Wolbachia bacteria from arthropods. Symbiosis. 2014;62(1):1-15. doi: 10.1007/s13199-014-0267-1. 